\title{
Reading aloud: The cumulative lexical interference effect
}

\author{
Claudio Mulatti • Francesca Peressotti • Remo Job • \\ Steven Saunders • Max Coltheart
}

Published online: 24 May 2012

(C) Psychonomic Society, Inc. 2012

\begin{abstract}
Picture naming shows a cumulative semantic interference effect: Latency for naming a target picture increases as a function of the number of pictures semantically similar to the target that have previously been named (Howard, Nickels, Coltheart, \& Cole-Virtue, Cognition 100:464-482, 2006). Howard and colleagues, and also Oppenheim, Dell, and Schwartz (Cognition 114:227-252, 2010), argued that this occurs because of the joint presence in the picture-naming system of three critical properties: shared activation, priming, and competition. They also discussed the possibility that whenever any cognitive system possesses these three properties, a cumulative similaritybased interference effect from repeated use of that cognitive system will occur. We investigated this possibility by looking for a cumulative lexical interference effect when the task is reading aloud: Will the latency of reading a target word aloud increase as a function of the number of words orthographically/phonologically similar to the target that have previously been read aloud? We found that this was so. This supports the general idea that cumulative similarity-based interference effects will arise whenever any cognitive system that possesses the three key properties of shared activation, priming, and competition is repeatedly used.
\end{abstract}

Electronic supplementary material The online version of this article (doi:10.3758/s13423-012-0269-z) contains supplementary material, which is available to authorized users.

C. Mulatti $(\bowtie) \cdot$ F. Peressotti

DPSS - Università degli Studi di Padova,

Via Venezia, 8,

35131 Padova, Italia

e-mail: claudio.mulatti@unipd.it

R. Job

DiSCoF - Università degli Studi di Trento,

Rovereto, Italia

S. Saunders $\cdot$ M. Coltheart

CCD - Macquarie University,

Sydney, Australia
Keywords Reading · Word production · Computational modeling

A cumulative semantic interference effect upon word production was reported by Howard, Nickels, Coltheart, and Cole-Virtue (2006). They presented participants with pictures to be named. The pictures were drawn from several semantic categories (animals, fruit, vehicles, etc.). Picture-naming reaction times (RTs) increased linearly as a function of the number of previously named pictures in that category: a cumulative semantic interference effect. Of particular note is the finding that the number of items intervening between two exemplars of the same category did not modulate the interference effect. For example, given the picture sequence pig, house, car, sheep, RTs for sheep would be longer than those for pig, regardless of the number of unrelated interspersed items. Navarrete, Mahon, and Caramazza (2010) replicated this effect. It might be thought of as related to the general phenomenon of retrieval-induced forgetting (Brown, 1981).

How might this effect be explained? And might we learn from it something interesting about the mechanisms of speech production? Howard et al. (2006) and Oppenheim, Dell, and Schwartz (2010), employing the same proposed functional architecture of the picturenaming and speech production system, suggested that the effect arises as a joint consequence of three properties of the cognitive system used in picture naming, these properties being shared activation, priming, and competition.

\section{Shared activation}

These authors argued that the semantic system possesses the property of shared activation. By this is meant the idea that 
when the semantic representation of a particular word-say, cat - is activated, the semantic representations of semantically similar words, such as $d o g$, will also be activated. If elements of the semantic system are atomic units representing individual concepts (compositional semantics), the property of shared activation could be implemented by an associative network in the semantic system linking semantically related units. If, instead, the elements of the semantic system are feature units, each representing a particular semantic feature such as + animate (decompositional semantics), then the property of shared activation would arise simply because the set of semantic features activated by any word or picture would partly overlap with the set of semantic features characterizing the meaning of any semantically related word or picture. On either view, activation of any semantic representation will result in the semantic representations of semantically related concepts also being activated. That is what is referred to as shared activation.

\section{Priming}

These authors also proposed that any activation/retrieval of a phonological lexical representation (as occurs, e.g., in picture naming) facilitates subsequent activation/retrieval of that representation. This is priming, which Howard et al. (2006) conceptualized as strengthening of item-specific links from the semantic system to the phonological lexicon. An alternative conceptualization of priming, which was not undertaken by these authors, sees the phenomenon as a saving effect: Some of the activation generated by the processing of the prime remains in the lexical representation, so that the processing of the target is facilitated.

\section{Competition}

The property of shared activation means that a word or picture will activate the semantics not only of itself, but also of concepts semantically similar to it. Given that there are itemspecific connections from semantics to the phonological lexicon, it follows that when a word or picture is presented, multiple competing entries in the phonological lexicon will be activated. It will, therefore, be necessary to ensure that the correct one among these competing multiple entries is selected for production. Howard et al. (2006) and Oppenheim et al. (2010) offered different accounts of the specific mechanism by which such competition is resolved (we take this issue up in the Discussion section), but those authors agreed that the system used for picture naming possesses the property of competition (as well as the other two properties just discussed, priming and shared activation) - for example, "Lexical retrieval leads to lexical learning. The light side of learning is well known. Retrieving the same word again becomes faster and more accurate. But learning also has a dark, competitive, side that hinders the subsequent retrieval of semantically related words" (Oppenheim et al., 2010, p. 247).

\section{How general might cumulative interference effects be?}

Both Howard et al. (2006) and Oppenheim et al. (2010) considered the idea that the three processing properties discussed above might well also characterize cognitive systems other than the visual/semantic/phonological system required for picture naming. Given this, if these properties are considered sufficient to generate cumulative interference effects whenever all three of them are present, one might observe cumulative interference effects in domains other than semantics: "We can identify cumulative semantic interference with a more general theme in the way the mind operates, independent of the particular types of representations in use" (Oppenheim et al., 2010, p. 250).

Consider the task of reading words aloud. There are good reasons to believe that the system used for reading words aloud exhibits the property of shared activation. Evidence for this view comes from the orthographic neighborhood density $(N)$ effect. $N$ refers to the number of words created by changing each letter in a stimulus, one at a time (Coltheart, Davelaar, Jonasson, \& Besner, 1977). In the case of reading aloud, as $N$ increases, the time taken to read aloud low-frequency words decreases (Andrews, 1992; McCann \& Besner, 1987; see also Mulatti, Reynolds, \& Besner, 2006, for an interpretation of $N$ effects in terms of phonological, rather than orthographic, shared activation). The conclusion here is that the $N$ effect on reading aloud arises from the coactivation (i.e., shared activation), within the lexical system, of lexical representations of words orthographically or phonologically similar to the actual stimulus.

Evidence of priming in reading-aloud tasks was provided by Scarborough, Cortese, and Scarborough (1977), who reported faster reading aloud for repeated items relative to novel items, even over lags greater than 100 intervening items (in their Experiment 4, they reported repetition benefit over a lag of 2 days). The same finding was reported by Visser and Besner (2001). This long-lag repetition benefit strongly suggests that in the reading-aloud system, the processing of a word primes its future retrieval(s). This might be interpreted theoretically as arising because, when a link from a word's entry in the orthographic lexicon to its entry in the phonological lexicon is used for reading aloud, that link is strengthened

Evidence that representations compete in reading comes from the study of nonword reading aloud. RTs to nonwords containing multiletter graphemes-that is, graphemes 
whose pronunciation depends on the processing of more than one letter (e.g., $\mathrm{PH} \rightarrow / \mathrm{f} /$ ) - are longer than RTs to nonwords that do not contain such graphemes: the whammy effect (Rastle \& Coltheart, 1998). It is explained in terms of competition between the phoneme activated by the first letters comprised in the multiletter grapheme (e.g., $\mathrm{P} \rightarrow / \mathrm{p} /$ ) and the phoneme activated by the multiletter grapheme itself (e.g., $\mathrm{PH} \rightarrow / \mathrm{f} /$ ). It is because there is competition at the phoneme level that the whammy effect arises.

So it is plausible to suggest that three properties implicated in the cumulative semantic interference effect according to Howard et al. (2006) and Oppenheim et al. (2010) also characterize the lexical system people use for reading words aloud. Hence, one might expect to observe a cumulative lexical interference effect in reading aloud, analogous to the cumulative semantic interference effect in picture naming. Our study sought to determine whether there is such a cumulative lexical interference effect.

We presented Italian participants with a list of Italian words to be read aloud. Words were drawn from 12 orthographic categories (e.g., the category -ana was composed of rana [frog], tana [den], vana [vain], sana [healthy], and lana [wool]). If the reading system exhibits cumulative costs of orthographic similarity, RTs will increase monotonically as a function of the number of previously read words in that orthographic category. For example, in the sequence rana, rate, coda, tana, gelo, lana, RTs for tana should be longer than those for rana, and RTs for lana longer than those for tana.

\section{Experiment}

Method

Participants Twenty students at the Università degli Studi di Padova with normal or corrected to normal vision participated.

Materials Sixty 4-letter Italian words were selected. These belonged to 12 different orthographic categories, where a category is defined as a set of words with the same ending (i.e., -ana, -ara, -aso, -elo, -ena, -ero, -ino, -olo, -ono, -oro, oto, -rto). Each category was composed of five items (e.g., the category -elo was composed of velo [veil], telo [length of cloth], melo [apple tree], gelo [frost], pelo [hair]). In addition, thirty-four 4-letter words were selected as fillers, with the constraint that none was an orthographic neighbor of the experimental words.

Design The 94 words were randomly inserted into a sequence, with the following constraints. Words from each orthographic category were separated by two, four, six, or eight intervening items. Each lag value (i.e., 2, 4, 6, and 8) was equally represented at each ordinal position (i.e., each lag was presented a total of three times at each of the ordinal positions, from 2 to 5). Filler items and categories in the sequence were randomly assigned. The first six items of the sequence were fillers. This same procedure was repeated nine times, giving 10 experimental sequences. In the 10 experimental sequences, a specific category was never presented at the same sequence position. The five items of each category occurred equally at each ordinal position within category. Participants were presented with one sequence. Each sequence was presented twice across participants, and assignment of participants to sequences was counterbalanced across participants.

Apparatus and procedure Participants were asked to read the words aloud as quickly and accurately as possible. Each trial involved the following events. A fixation cross appeared in the center of the screen for $494 \mathrm{~ms}$, followed by a blank interval of $106 \mathrm{~ms}$. The word then appeared and remained visible until participants responded or $3 \mathrm{~s}$ elapsed. The intertrial interval was set to $800 \mathrm{~ms}$. Stimulus presentation and data collection were controlled by software developed in E-Prime and running on a computer to which a voice key (Serial Response Box, Psychology Software Tools Inc.) was connected.

\section{Results}

Reading errors $(0.9 \%)$ and voice key failures $(1.1 \%)$ were excluded from RT analyses. Errors were few and not further analyzed. Figure 1 plots RTs according to conditions.

Our design minimized but did not eliminate a confounding between ordinal position within category (OPWC; five levels: 1-5) and serial position of the item in the whole experiment (hereafter, position within list; PWL). To control for this, we used linear mixed effects modeling (Baayen, 2008). Such modeling is based on single-trial data, rather

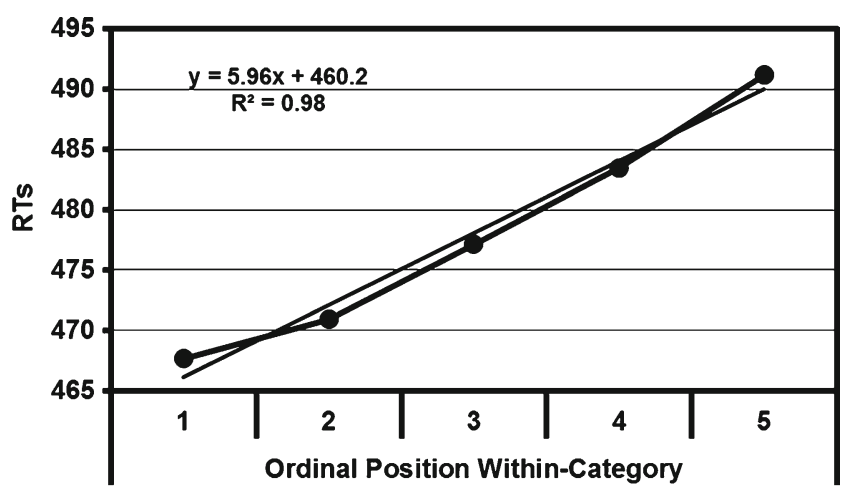

Fig. 1 Mean reaction times (RTs) according to the ordinal position of items within orthographic category 
than on averaged data, allowing the estimation of the effects of both OPWC and lag once PWL effects are controlled for. First, we defined a model $(\mathrm{ml})$ with participants and items as random factors, and PWL as a fixed factor along with its interaction with participants (i.e. the PWL effects were allowed to vary between participants). Then we defined a second model (m2), adding OPWC as a fixed factor. A formal comparison of $\mathrm{m} 1$ and $\mathrm{m} 2$ (namely, a loglikelihood test) showed a significant improvement in model fit, $\chi^{2}(1)=4.7, p<.05$. In model $\mathrm{m} 3$, we tested for the effect of lag by adding it to $\mathrm{m} 2$ as a fixed factor: The increase in the model fit was not significant, $\chi^{2}(1)=0.16$, $p>.69$, which suggests that lag has no significant effects. Chi-square comparison also revealed that a model, $\mathrm{m} 4$, with the interaction between lag and OPWC did not provide a better fit to the data than either $\mathrm{m} 2$ or $\mathrm{m} 3$, which implies that the interaction was not significant. The evaluation of fixed effects in the best-fitting model, $\mathrm{m} 2$, and in the full additive model, $\mathrm{m} 3$, is reported in Table 1 .

A further analysis was performed on the best-fitting model, $\mathrm{m} 2$, to test linear, quadratic, and cubic components of the OPWC effect. Whereas the linear component was significant, $t(1180)=2.17, \mathrm{p}<.05$, neither the quadratic component, $|t|<1$, nor the cubic component, $|t|<1$, proved significant. ${ }^{1}$ Thus, analyses with the linear mixed model showed a significant linear effect of OPWC and that RTs are not affected by the number of items intervening between two members of the same orthographic category - that is, no effect of lag. ${ }^{2}$ Thus, our reading-aloud results exactly matched the picture-naming results of Howard et al. (2006).

\section{Discussion}

This experiment is the first to examine the extent to which there are cumulative effects of lexical competitors when the task is reading words aloud. Word reading aloud latency is slowed by an additional $6 \mathrm{~ms}$ for each preceding lexically similar word. In addition, the number of intervening items (whether two, four, six, or eight) between two exemplars of the same orthographic category is irrelevant to the effect.

\footnotetext{
${ }^{1}$ Degrees of freedom are difficult to compute in linear mixed effects modeling, and thus significance of factors is usually estimated via pMCMC values ( $p$-values calculated with Markovian-chain Monte Carlo), rather than via $t$ statistics. However, pMCMC values cannot be computed if the model includes an interaction between a fixed and a random effect, because MCMC sampling is not yet implemented for models with random correlation parameters. Baayen (2008) suggests that "an upper bound for the degrees of freedom for the t-tests can be obtained by taking the number of observations and subtracting the number of fixed-effects parameters, p. 248." We have followed his suggestion to analyze the data.

${ }^{2}$ The analyses with $\log$ transformed data yield the same pattern of result as that obtained with RTs.
}

Table 1 Fixed effects in linear mixed models $\mathrm{m} 2$ and $\mathrm{m} 3$. PWL $=$ position within-list; OPWC $=$ ordinal position within-category; Lag = number of items interspersed between two exemplars of the same orthographic category

\begin{tabular}{|c|c|c|c|c|c|}
\hline \multicolumn{2}{|c|}{ Models } & \multicolumn{3}{|c|}{ Fixed effects } & \multirow{2}{*}{$\begin{array}{l}\text { Highest correlation } \\
\text { among fixed effects }\end{array}$} \\
\hline & & PWL & OPWC & Lag & \\
\hline \multirow[t]{3}{*}{$\mathrm{m} 2$} & $\beta$ & 0.47 & 3.16 & & \multirow[t]{3}{*}{ Intercept : PWL .481 } \\
\hline & $\mathrm{t}(1180)$ & 3.1 & 2.2 & & \\
\hline & $\mathrm{p}$ & $<.005$ & $<.05$ & & \\
\hline \multirow[t]{3}{*}{$\mathrm{m} 3$} & $\beta$ & 0.47 & 3.47 & -0.31 & \multirow[t]{3}{*}{ Intercept : PWL .482 } \\
\hline & $\mathrm{t}(1179)$ & 3.1 & 2.1 & -0.4 & \\
\hline & $\mathrm{p}$ & $<.005$ & $<.05$ & $>.69$ & \\
\hline
\end{tabular}

This cumulative lexical interference effect exactly parallels the cumulative semantic similarity effect seen when the task is picture naming. Its existence lends support to the general idea that whenever a task uses a cognitive system that possesses the properties of shared activation, priming, and competition, that task will exhibit a cumulative similaritybased interference effect.

How is competition implemented? Although Howard et al. (2006) and Oppenheim et al. (2010) agreed that the cumulative semantic interference effect is due to the joint effects of these three properties, they disagreed about how one of these properties - competition - is implemented in the system used for picture-naming. According to Howard and colleagues, competition arises because of lateral inhibition within the phonological lexicon. Oppenheim and colleagues denied that there is such lateral inhibition in the phonological lexicon and implemented competition in a different way, proposing that, when the production of a word strengthens the link between that word's semantic representation and its entry in the phonological lexicon (due to the system property of priming), at the same time the links between semantics and phonology for semantically related words are weakened (this is referred to as competitive learning). Thus, naming a picture of a dog will be slower if one has named a picture of a cat a few trials before, because the semantics-tophonology link for $d o g$ has been weakened; that is where the semantic interference comes from. The more animals that have been named before $d o g$ is presented, the weaker the link for $d o g$ will have become; that is why the semantic interference effect is cumulative.

Navarrete et al. (2010) investigated these contrasting ways of implementing competition. They reported the results of an experiment involving spoken production of nouns preceded by their gender-appropriate determiners. The stimuli were printed nouns. The experiment was run in Italian and German. The point of this experiment was that in these two languages, grammatical gender is a property of 
Fig. 2 A minimal architecture for picture naming and lexical reading

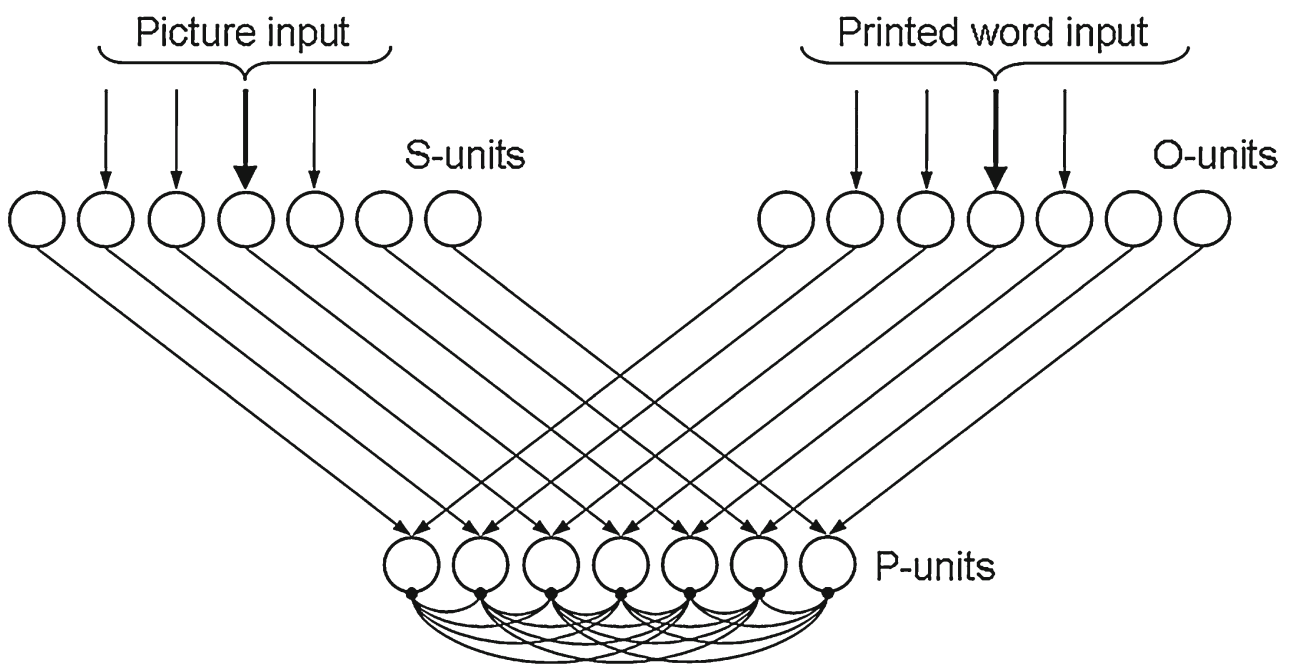

lexical representations, which means that to perform this reading-aloud task correctly, it is necessary to access the lexical node for each word (if the task had simply been to read aloud each bare noun, given the very regular spellingto-sound correspondences of German and Italian, the task could have achieved largely or entirely nonlexically). If there is lateral inhibition at the lexical level and if that lateral inhibition is implicated in the cumulative semantic interference effect, the cumulative semantic interference effect should be observed in this determiner + noun readingaloud task. However, there was no such effect in this experiment. The authors argued that the absence of the effect here represented evidence that there is no lateral inhibition within the lexical level and, so, took their results as support for the idea that competition should be implemented in the way proposed by Oppenheim et al. (2010).

Now, although this task arguably does require access to the phonological lexicon, it does not require access to the semantic system and, so, does not necessarily produce shared activation; and if there is no shared activation influencing task performance, then, on the account offered by Howard and colleagues, no cumulative semantic interference would be expected. Hence, Navarrete et al. (2010) needed to argue that activation of the semantic system does occur as part of the determiner + noun reading-aloud task. Support for this comes from the results of a study by Damian, Vigliocco, and Levelt (2001), who reported that cumulative semantic effects occur with a cyclic semantic blocking experiment using the determiner + noun reading aloud task. But this raises the following issue.

Given the account offered by Oppenheim et al. (2010) of the cumulative semantic interference effect, how is the absence of this effect in the determiner + noun reading-aloud task to be explained? Since that task involves activation of a word and its semantic associates at the semantic level and, hence, activation of the semantic-phonological links for these words, why is there no competitive learning that weakens the nontarget links (as happens when the task is picture naming and produces the cumulative semantic interference effect in that task)? Navarrete et al. (2010) respond to this issue by proposing that there are direct links from the orthographic forms of words to their phonological forms, as a consequence of which "the identity of the target word is never in doubt as it is given by the stimulus input, p. 284"; so competitors are not a threat; so a mechanism for weakening competitors is not needed. However, evidence from the literature on reading indicates that when a word is visually presented, there is activation of its orthographic/ phonological neighbors (Andrews, 1992; McCann \& Besner, 1987), so it is difficult to argue that the direct pathway from orthography to phonology activates only a single item and not any of its competitors. Moreover, if, in the determiner + noun reading aloud task, there is no weakening of the semantic competitors, how can the cumulative semantic interference found in the cyclic semantic blocking paradigm by Damian et al. (2001) be accounted for?

The picture gets even more complicated if we consider the study of Vitkovitch, Cooper-Pye, and Ali (2010; see also Tree \& Hirsh, 2003). They presented their subjects with two blocks of stimuli. In the first block, the stimuli were words; in the second block, the stimuli were pictures. Subjects had to read the word aloud (the determiner was not required) and to name the pictures. Half of the pictures in the second block were semantically related to the words in the first block; the remaining pictures bore no relation to preceding words. Vitkovitch et al. showed that RTs to semantically related pictures were longer than RTs to unrelated pictures; that is, a semantic interference effect was observed. So, whereas Navarrete et al. (2010) showed that determiner + noun reading aloud does not interfere with either the subsequent determiner + noun reading of a semantically related word or with the subsequent naming of a semantically related picture, Vitkovitch et al. showed that the reading aloud of a word interferes with the subsequent naming of a 
semantically related picture. It is important to note that whereas the determiner + noun reading aloud of semantically related words in a cyclic blocking paradigm does result in interference, the reading aloud (no determiner) of semantically related words in a cyclic blocking paradigm does not result in interference (Damian et al., 2001).

So we concur with the view offered by Navarrete et al. (2010, p. 287) that there are some unresolved questions here that require further empirical work for their resolution. Thus, while it seems reasonable that both the picture-naming system and the reading-aloud system possess the property of competition, we cannot be confident in asserting just how this property is implemented in these systems: that is, whether it is within the phonological lexicon, in the mapping between the semantic and orthographic representations and the phonological representations, or even somewhere else.

Modeling We conclude by proposing a generalization of the model architecture for picture naming offered by Howard et al. (2006, p. 472, Fig. 2; the same levels exist in the model of Oppenheim et al., 2010) by adding to it a lexical reading route that converges on the same phonological lexical output units (this generalized architecture is shown in Fig. 2). This generalized architecture possesses throughout the three critical properties of shared activation (which can be semantic or lexical), competition, and priming, and it is the possession of these three properties that is responsible for cumulative semantic or lexical interference effects.

The O-unit to P-unit pathway in Fig. 2 corresponds to the direct route from orthographic lexicon to phonological lexicon in the dual-route cascaded (DRC) computational model of visual word recognition and reading aloud (Coltheart, Rastle, Perry, Langdon, \& Ziegler, 2001). That model already possesses the properties of shared activation and competition. The property of priming is simple to add: All that needs to be done is to modify the model by arranging for the connection between a word's entry in the orthographic lexicon and its entry in the phonological lexicon to be strengthened whenever that word is read aloud by the model. Preliminary simulations with the DRC model modified in this way have succeeded in producing a cumulative lexical interference effect on the model's reading-aloud RT (see the online supplement). In this preliminary work, competition arises in the model because of lateral inhibition within the phonological lexicon (and within the phoneme system). But one could, instead, implement lexical competition in the model in the way envisaged by Oppenheim et al. (2010): Whenever a word is read aloud, its connection from orthographic lexicon to phonological lexicon is strengthened, but also the connections of its competing neighbors are weakened. Comparisons of these two kinds of simulations could contribute to resolving the issue of how to implement the competition that is part of the cause of cumulative similarity-based interference effects.

\section{References}

Andrews, S. (1992). Frequency and neighborhood effects on lexical access: Lexical similarity or orthographic redundancy? Journal of Experimental Psychology: Learning, Memory, and Cognition, 18, 234-254.

Baayen, H. (2008). Analyzing linguistic data: A practical introduction to statistics. Cambridge: Cambridge University Press.

Brown, A. S. (1981). Inhibition in cued retrieval. Journal of Experimental Psychology: Human Learning and Memory, 7, 204-215.

Coltheart, M., Davelaar, E., Jonasson, J. T., \& Besner, D. (1977). Access to the internal lexicon. In S. Dornic (Ed.), Attention and performance VI (pp. 535-555). Hillsdale: Erlbaum.

Coltheart, M., Rastle, K., Perry, C., Langdon, R., \& Ziegler, J. (2001). DRC: A dual route cascaded model of visual word recognition and reading aloud. Psychological Review, 108, 204-256.

Damian, M. F., Vigliocco, G., \& Levelt, W. J. (2001). Effects of semantic context in the naming of pictures and words. Cognition, 81, B77-B86.

Howard, D., Nickels, L., Coltheart, M., \& Cole-Virtue, J. (2006). Cumulative semantic inhibition in picture naming: Experimental and computational studies. Cognition, 100, 464-482.

McCann, R. S., \& Besner, D. (1987). Reading pseudohomophones: Implications for models of pronunciation assembly and the locus of word frequency effects in naming. Journal of Experimental Psychology: Human Perception and Performance, 13, 14-24.

Mulatti, C., Reynolds, M. G., \& Besner, D. (2006). Neighborhood effects in reading aloud: New findings and new challenges for computational models. Journal of Experimental Psychology: Human Perception and Performance, 32, 799-810.

Navarrete, E., Mahon, B. Z., \& Caramazza, A. (2010). The cumulative semantic cost does not reflect lexical selection by competition. Acta Psychologica, 134, 279-289.

Oppenheim, G. M., Dell, G. S., \& Schwartz, M. F. (2010). The dark side of incremental learning: A model of cumulative semantic interference during lexical access in speech production. Cognition, 114, 227-252.

Rastle, K., \& Coltheart, M. (1998). Whammies and double whammies: The effect of length on nonword reading. Psychonomic Bulletin \& Review, 5, 277-282.

Scarborough, D. L., Cortese, C., \& Scarborough, H. S. (1977). Frequency and repetition effects in lexical memory. Journal of Experimental Psychology: Human Perception and Performance, 3, $1-17$.

Tree, J. J., \& Hirsh, K. W. (2003). Sometimes faster, sometimes, slower: Associative and competitor priming in picture naming with young and elderly participants. Journal of Neurolinguistics, 16, 489-514.

Visser, T. A. W., \& Besner, D. (2001). On the dominance of wholeword knowledge in reading aloud. Psychonomic Bulletin \& Review, 8, 560-567.

Vitkovitch, M., Cooper-Pye, E., \& Ali, L. (2010). The long and the short of it! Naming a set of prime words before a set of related picture targets at two different intertrial intervals. European Journal of Cognitive Psychology, 22, 161-171. 